\title{
CALCULATING DISCRIMINANTS BY HIGHER DIRECT IMAGES
}

\author{
JERZY WEYMAN
}

\begin{abstract}
The author uses the homological algebra to construct for any line bundle $\mathscr{L}$ on a nonsingular projective variety $X$ the complex $\mathbb{F}(\mathscr{L})$ whose determinant is equal to the equation of the dual variety $X^{\vee}$. This generalizes the Cayley-Koszul complexes defined by Gelfand, Kapranov and Zelevinski. The formulas for the codimension and degree of $X^{\vee}$ in terms of complexes $\mathbb{F}(\mathscr{L})$ are given. In the second part of the article the general technique is applied to classical discriminants and hyperdeterminants.
\end{abstract}

This paper consists of two parts. In the first part ( $\S 1$ and 2) we give the method for calculating generalized discriminants considered by Gelfand, Kapranov and Zelevinski (cf. [G-K-Z]). The generalized discriminant is defined for any nonsingular projective variety $X$ as the defining equation of the dual variety $X^{\vee}$. The method for calculating these equations introduced here uses the conormal construction and the technique of higher direct images. For each line bundle $\mathscr{L}$ on $X$ we define the canonical complex $\mathbb{F}(\mathscr{L})$, obtained by pushing down the twisted Koszul resolutions associated to the canonical desingularisation of the dual variety $X^{\vee}$. The complex $\mathbb{F}(\mathscr{L})$ allows to calculate explicitly the degree of the discriminant and give the expression for it in terms of Buchsbaum-Eisenbud multipliers associated to $\mathbb{F}(\mathscr{L})$.

The general technique is described in $\S 1$ where all general formulas are proved. Section 2 deals with the case of $G$-discriminants. They occur when the nonsingular variety $X$ is the $G$-orbit of the highest weight vector in the irreducible representation of the reductive group $G$.

In the second part ( $\S 3$ and 4 ) we investigate the determinantal expressions of the discriminants. They arise naturally in the context of the complexes $\mathbb{F}(\mathscr{L})$. If the complex $\mathbb{F}(\mathscr{L})$ has only two terms, it reduces to a matrix. In such cases the discriminant becomes the determinant of this matrix.

Section 3 deals with the case of the discriminant of a homogeneous polynomial of degree $m$. Classically such formulas were known for the case of two variables (Sylvester and Bezout formulas) and three variables, which is treated in the book of Salmon [S]. In $\S 3$ we give a list of such formulas. In the case of two variables this list includes the Sylvester expression ( $\$ 3.2, m$ arbitrary, $a=-1$ ) and Bezout expression ( $\$ 3.2, m$ arbitrary, $a=m-2$ ). Moreover, these formulas appear as the extreme cases of the whole series of determinantal expressions of similar type. Section 3.3 deals with the case of three variables.

Received by the editors March 27, 1992 and, in revised form, October 5, 1992.

1991 Mathematics Subject Classification. Primary 13D25, 14N05; Secondary 13D02, 14M15, $15 \mathrm{~A} 72$. 
Here we also give the whole series of determinantal formulas for the discriminant, including the one given by Salmon ( $\$ 3.3, m$ arbitrary, $a=2 m-3$ ). The most interesting is the fact that even for a polynomial in four variables of degree $m$ there always exists a determinantal expression for its discriminant. It is described in $\S 3.4$. This fact was not known to classics. The rest of $\S 3$ deals with some exceptional cases in 5 and 6 variables where the determinantal formulas for discriminant still occur. We should notice that in this paper we describe only the "formats" of the determinants in question without giving the explicit matrix. We plan to give an explicit description of these matrices in a subsequent paper.

In $\S 4$ we consider the case of discriminants of multidimensional matrices (multilinear forms). This subject also goes back to Cayley, but was recently revived by Gelfand, Kapranov and Zelevinski (cf. [G-K-Z]).

Our method allows to recover the formulas given there in the boundary case (§4.3). One can also derive and generalize the formula for the discriminant of 3 dimensional matrix of the boundary format given by Bondal ( $\$ 4.3)$. Finally we give new formulas for subboundary case $(\$ 4.4)$ and show that the determinantal expression occur for relatively few formats of matrices $(\S 4.5)$.

In $\S 4.6$ we construct certain complex associated to the multilinear form $A$ which plays the role analogous to the Koszul complex of the Jacobian ideal of a homogeneous polynomial.

Section 4.7 is devoted to the exterior case, i.e. the case when the discriminant variety has codimension bigger than 1 . We find the generators of the defining ideal of this variety for $m \times 2 \times 2$ matrices $(m>3)$ and $m \times 3 \times 2$ matrices $(m>4)$.

\section{The Main CONSTRUCtion}

1.1. Let $V$ be a finite dimensional vector space over $\mathbb{C}$, and let $X \subset \mathbb{P}\left(V^{*}\right)$ be a smooth projective variety of dimension $n$. Let $Y \subset V^{*}$ be the cone over $X$. Let us assume that $X$ is linearly normal and nondegenerate, i.e. the natural map $V \rightarrow H^{0}\left(X, \mathscr{O}_{X}\right)$ is an isomorphism.

We define $X^{\vee} \subset \mathbb{P}(V)$ to be the projectively dual variety to $X$. By definition $X^{\vee}$ consists of hyperplanes in $\mathbb{P}\left(V^{*}\right)$ tangent to $X$ at some point of $X$. If $\operatorname{codim} X^{\vee}=1$, then the homogeneous polynomial $\Delta_{X}$ vanishing on $X^{\vee}$ is called the discriminant of $X$. The polynomial $\Delta_{X}$ is defined up to a nonzero constant. If $\operatorname{codim} X^{\vee}>1$, we define $\Delta_{X}$ to be a nonzero constant. We denote by $Y^{\vee}$ the cone over $Y$. This notion was recently considered by Gelfand, Kapranov and Zelevinski [G-K-Z].

1.2. The main idea of this paper comes from the observation that $X^{\vee}$ is the image of certain vector bundle. Let us define the subvariety $Z^{\prime}$ of $X \times \mathbb{P}(V)$,

$$
Z^{\prime}=\{(x, f) \in X \times \mathbb{P}(V) \mid f \text { is tangent to } X \text { at } x\} .
$$

It is clear that the first projection $p^{\prime}: Z^{\prime} \rightarrow X$ makes $Z$ a projectivized vector bundle over $X$, and that the image of the second projection $q^{\prime}: Z^{\prime} \rightarrow \mathbb{P}(V)$ is equal to $X^{\vee}$. We denote by $Z$ the cone over $Z^{\prime}$, i.e.,

$$
Z=\{(x, f) \in X \times V \mid f \text { is tangent to } X \text { at } x\} \text {. }
$$

The first projection $p: Z \rightarrow X$ makes $Z$ a vector bundle over $X$, and the image of the second projection $q: Z \rightarrow V$ is equal to $Y^{\vee}$. Let us consider the 
locally free resolution of $\mathscr{O}_{Z}$ as $\mathscr{O}_{X \times V}$-module. It is a Koszul complex on the vector bundle $\mathscr{S}$ on $X \times V$. In fact it is easy to see that $\mathscr{S}$ equals to $p^{*}(\xi)$ where $\xi$ is the natural bundle over $X$ which satisfies the exact sequence

$$
0 \rightarrow \mathscr{O}_{X}(-1) \rightarrow \xi \rightarrow T(X)(-1) \rightarrow 0 .
$$

Here $T(X)$ denotes the tangent bundle on $X$. There is a simple way to describe $\xi(1)$ geometrically. For any $x \in X$ the fibre $\xi(1)_{x}$ is the tangent space to the cone $Y$ over $X$.

Thus we have the exact complex of locally free modules on $X \times \mathbb{P}(V)$.

$$
\bigwedge \cdot(\mathscr{S}) \rightarrow \mathscr{O}_{Z} \rightarrow 0
$$

Let $R=\operatorname{Sym}\left(V^{*}\right)$ denote the polynomial ring of homogeneous functions on $\mathbb{P}(V)$. The following proposition is an immediate consequence of the Theorem 2.1 from [W].

1.3. Proposition. There exists the complex

$$
\mathbb{F}: \cdots \rightarrow F_{i} \rightarrow F_{i-1} \rightarrow \cdots
$$

of free R-modules, where

$$
F_{i}=\bigoplus_{j \geq 0} \mathscr{R}^{j} q_{*}\left(\bigwedge^{i+j} \mathscr{S}\right) \otimes_{\mathbb{C}} R=\bigoplus_{j \geq 0} H^{j}\left(X, \bigwedge^{i+j} \xi\right) \otimes_{\mathbb{C}} R,
$$

whose homology equals

$$
H_{-i}(\mathbb{F})=\mathscr{R}^{i} q_{*}\left(\mathscr{O}_{Z}\right) .
$$

We want to show that the properties of the discriminant (its degree and the expression for it) follow from the knowledge of the complex $\mathbb{F}$. The expressions we get in this way are similar to those obtained by Gelfand, Kapranov and Zelevinski using Cayley-Koszul complexes, but more convenient, because we have the precise knowledge of the homology of $\mathbb{F}$. Also the use of BuchsbaumEisenbud structure theorem makes the notion of the determinant of a complex used in [G-K-Z] much more precise.

1.4. Let us notice that $\operatorname{dim} Z=\operatorname{dim} V-1$. We distinguish three cases:

(1a) the map $Z \rightarrow Y^{\vee}$ is a desingularisation.

(1b) $\operatorname{codim} Y^{\vee}=1$, the map $Z \rightarrow Y^{\vee}$ is generically a covering of degree $d$, $d>1$

(2) $\operatorname{codim} Y^{\vee}>1$.

It is not difficult to show (compare [Ka]) that the case (1b)) in fact cannot occur. We want to see how to distinguish between the cases (1) and (2) using the complex $\mathbb{F}$. First of all let us notice that the modules $\mathscr{R}^{i} q_{*}\left(\mathscr{O}_{Z}\right)$ for $i>0$ have the support of codimension at least 2 . It is clear in case (2). In case (1) we notice that the stalks of sheaves associated to these modules are 0 at the points where the fibers of $p$ have dimension 0 . Thus we can consider the graded Hilbert function

$$
P(\mathbb{F}, t)=\sum_{i, j \geq 0}(-1)^{i} t^{i+j} \operatorname{dim} H^{j}\left(X, \bigwedge^{i+j} \xi\right)(1-t)^{-\operatorname{dim} V}
$$


of the complex $\mathbb{F}$. Let us write $P(\mathbb{F}, t)=\sum P(a) t^{a}$. Then for $a>>P(a)$ is a polynomial in $a$. Since the function $P(\mathbb{F}, t)$ is the alternating sum of Hilbert functions of $\mathscr{R}^{i} q_{*}\left(\mathscr{O}_{Z}\right), P(a)$ is a polynomial in $a$ of degree $\leq \operatorname{dim} V-1$.

Let us analyse the support of the modules $\mathscr{R}^{i} q_{*}\left(\mathscr{O}_{Z}\right)$. For $i>0$ these modules have the support of codimension $\geq 2$. The module $\mathscr{R}^{0} q_{*}\left(\mathscr{O}_{Z}\right)$ is the module of rank 1 supported in $Y^{\vee}$ in the case (1), and it is supported in codimension 2 in the case (2).

Thus the coefficient of $P(a)$ in degree $\operatorname{dim} V-1$ (divided by $(\operatorname{dim} V-1)$ !) equals to 0 in the case (2), and to $\operatorname{deg} \Delta_{X}$ in the case (1a). The standard calculation shows that this coefficient equals

$$
e(\mathbb{F})=\sum_{i, j \geq 0}(-1)^{i+1}(i+j) \operatorname{dim} H^{j}\left(X, \bigwedge^{i+j} \xi\right) .
$$

This means we have proved

1.5. Proposition. The number

$$
e(\mathbb{F})=\sum_{i, j \geq 0}(-1)^{i+1}(i+j) \operatorname{dim} H^{j}\left(X, \bigwedge^{i+j} \xi\right)
$$

is equal to $\operatorname{deg} \Delta_{X}$ in the case (1a), and to 0 in the case (2).

1.6. One can get another expression for $\operatorname{deg} \Delta_{X}$ in terms of Chern classes. It comes from calculating the class of $Z^{\prime}$ in the Chow ring of $X \times \mathbb{P}\left(V^{*}\right)$ and pushing down. This formula is discussed for example in the article of Kleiman $[\mathrm{K}]$. The Chern classes approach allows also to calculate the codimension of $Y^{\vee}$. This was done by Holme in [Ho]. Our method also allows to express the codimension of $Y^{\vee}$ in a very nice way.

\subsection{Theorem. Codim $Y^{\vee}=\max \left\{i ; F_{i} \neq 0\right\}$.}

Proof. Let us consider the canonical sheaf $\omega_{Z}$. By the adjunction formula $[\mathrm{H}$, Proposition 11.8.20] $\omega_{Z}=\left.\omega_{X \times V}\right|_{Z} \otimes \Lambda^{n+1} \xi^{*}$. Since $V$ is just the affine space, $\omega_{X \times V}=p^{*} \omega_{X}$. Let us consider the resolution of $\omega_{Z}$ as $\mathscr{O}_{X \times V}$-module. It equals $p^{*}\left(\omega_{X} \otimes \Lambda^{n+1} \xi^{*}\right) \otimes \Lambda \cdot \mathscr{S}$. Pushing down this resolution we get the complex $D(\mathbb{F})$ of $R$-modules with the term

$$
D(\mathbb{F})_{i}=\bigoplus_{j \geq 0} H^{j}\left(X, \omega_{X} \otimes \bigwedge^{n+1-i-j} \xi^{*}\right)
$$

whose cohomology equals $H_{-i}(D(\mathbb{F}))=\mathscr{R}^{i} q_{*}\left(\omega_{Z}\right)$. By the GrauertRiemanschneider theorem $\mathscr{R}^{i} q_{*}\left(\omega_{Z}\right)=0$ for $i>\left(\operatorname{dim} Z-\operatorname{dim} Y^{\vee}\right)$. Since the differentials in $D(\mathbb{F})$ have the entries in the maximal ideal of $R$, this means that $D(\mathbb{F})_{i}=0$ for $i<\left(\operatorname{dim} Y^{\vee}-\operatorname{dim} Z\right)$. Now using the Serre duality on $X$ we see that $\mathbb{F}_{i}$ is the dual of $D(\mathbb{F})_{1-i}$. This means that $\mathbb{F}_{i}=0$ for $i>1-\operatorname{dim} Y^{\vee}+\operatorname{dim} Z=\operatorname{codim} Y^{\vee}$. To prove the theorem it is enough to show that for $i=\operatorname{dim} Z-\operatorname{dim} Y^{\vee}$, we have $\mathscr{R}^{i} q_{*}\left(\omega_{Z}\right) \neq 0$. However this is easy. Indeed, after shrinking $Y^{\vee}$ we may assume that $p$ is smooth, projective. Then the result follows from upper-semicontinuity theorem [H, III.12.11] and adjunction formula [H, II.8.20], since each fibre $Z_{y}$ is smooth of dimension $i$, so $H^{i}\left(Z_{y}, \omega_{Z_{y}}\right)$ is one-dimensional (hence nonzero) by Serre duality. 
1.8. Remark. Let $\mathscr{L}$ be a line bundle on $X$. Then we can consider the twisted version of the Proposition 1.3. It says that there exists a complex

$$
\mathbb{F}(\mathscr{L}): \cdots \rightarrow F_{i}(\mathscr{L}) \rightarrow F_{i-1}(\mathscr{L}) \rightarrow \cdots
$$

of free $R$-modules, where

(9)

$$
F_{i}(\mathscr{L})=\bigoplus_{j \geq 0} \mathscr{R}^{j} q_{*}\left(\bigwedge^{i+j} \mathscr{S} \otimes \mathscr{L}\right) \otimes_{\mathbb{C}} R=\bigoplus_{j \geq 0} H^{j}\left(X, \bigwedge^{i+j} \xi \otimes \mathscr{L}\right) \otimes_{\mathbb{C}} R,
$$

whose homology equals

$$
H_{-i}(\mathbb{F})=\mathscr{R}^{i} q_{*}\left(\mathscr{O}_{Z} \otimes \mathscr{L}\right) .
$$

The formula from Proposition (1.5) also generalizes to the twisted case.

Proposition. The number

$$
e(\mathbb{F}(\mathscr{L}))=\sum_{i, j \geq 0}(-1)^{i+1}(i+j) \operatorname{dim} H^{j}\left(X, \bigwedge^{i+j} \xi \otimes \mathscr{L}\right)
$$

is equal to $\operatorname{deg} \Delta_{X}$ in the case (1a), and to 0 in the case (2).

Proof. The same as the proof of Proposition 1.5.

1.9. Remark. The complexes $D(\mathbb{F}(\mathscr{L}))$ used in the proof of Theorem 1.7 are closely connected to the Cayley-Koszul complexes of Gelfand, Kapranov and Zelevinski. In order to obtain Cayley-Koszul complexes (in the case of toric varieties) they use the explicit acyclic resolutions of the exterior powers of the cotangent bundles. This means that our $D(\mathbb{F}(\mathscr{L}))$ is the $E^{1}$ term of the spectral sequence of the Cayley-Koszul complex. The formulas in [G-K-Z] aiso require twisting by a positive line bundle $\mathscr{O}(m)$ in order to get the vanishing of higher cohomology groups, so only the terms involving $H^{0}$ remain in the complex.

1.10. Let $Y$ be the cone over a nonsingular variety $X \subset \mathbb{P}\left(V^{*}\right)$. Let us consider the discriminant variety $Y^{\vee}$. We assume that the map $q: Z \rightarrow Y^{\vee}$ is a desingularisation, i.e. we are in the case (1a). This, by Theorem 1.7 is equivalent to the following condition:

(*) The module $F_{i}=0$ for $i>1$.

The condition $(*)$ is satisfied for most of the varieties $X$. Indeed, if the bundle $\mathscr{O}_{X}(1)$ is very positive, then by Serre theorem for all positive exterior powers of $\xi$ only the top cohomology group is nonzero.

Under the above assumption we will get the explicit expression for $\Delta_{X}$ in terms of Buchsbaum-Eisenbud multipliers. Let us recall the first structure theorem of Buchsbaum-Eisenbud [B-E, E-N].

\subsection{Theorem. Let $R$ be a commutative ring. Let}

$$
\mathbb{G}: 0 \rightarrow G_{n} \stackrel{d_{n}}{\longrightarrow} G_{n-1} \stackrel{d_{n-1}}{\longrightarrow} \cdots \stackrel{d_{2}}{\longrightarrow} G_{1} \stackrel{d_{0}}{\longrightarrow} G_{0}
$$

be a complex of finite dimensional free $R$-modules. Let us assume that $\mathbb{G}$ is acyclic in codimension 1 . Let $r_{m}=\operatorname{rank} d_{m}$. Then

(a) $r_{m}+r_{m+1}=\operatorname{dim} G_{m}$ for all $m, 1 \leq m \leq n$,

(b) there exists a unique sequence of maps $a_{m}: R \rightarrow \bigwedge^{r_{m}} G_{m-1} \quad(1 \leq m \leq$ $n)$, such that $a_{n}=\bigwedge^{r_{n}} d_{n}$, and the following diagrams commute: 


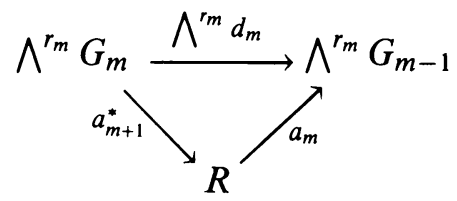

(here we identify $\Lambda^{r_{m}} G_{m}$ with $\Lambda^{r_{m}} G_{m}^{*}$ ).

Let us consider our complex

$$
\mathbb{F}: 0 \rightarrow F_{1} \stackrel{\left(d_{1}^{\prime}, y\right)}{\longrightarrow} F_{0}^{\prime} \oplus R \stackrel{d_{0}}{\longrightarrow} F_{-1} \rightarrow \cdots .
$$

In $F_{0}$ we split off the summand $R=H^{0}\left(X, \Lambda^{0} \xi\right) \otimes_{\mathbb{C}} R$. Since $\mathscr{R}^{0} q_{*}\left(\mathscr{O}_{\mathbf{Z}}\right)$ is the normalisation of the coordinate ring of $X^{\vee}$ [KKMS], we have the map of complexes

$$
\begin{array}{ll}
0 \longrightarrow & F_{1} \stackrel{\left(d_{1}^{\prime}, y\right)}{\longrightarrow} F_{0}^{\prime} \oplus R \stackrel{d_{0}}{\longrightarrow} F_{-1} \longrightarrow \cdots \\
& \uparrow_{u} \longrightarrow(0,1) \\
0 \longrightarrow & R \stackrel{\Delta_{X}}{\longrightarrow} \quad R
\end{array}
$$

It follows from 1.4 that the cone of this map is exact in codimension 1. This cone is homotopically equivalent to the following complex

$$
0 \rightarrow R \stackrel{u}{\longrightarrow} F_{1} \stackrel{d_{1}^{\prime}}{\longrightarrow} F_{0}^{\prime} \stackrel{d_{0}}{\longrightarrow} F_{-1} \rightarrow \cdots .
$$

Using Theorem 1.11 we deduce that $r=\operatorname{rank} d_{1}^{\prime}=\operatorname{dim} F_{1}-1$. This means that there exists a map $a: R \rightarrow \bigwedge^{r} F_{0}^{\prime}$, such that $r \times r$ minor $M_{j^{\wedge}, 1}$ of $d_{1}^{\prime}$ corresponding to the columns except the $j$ th one, and the rows from $I$, equals $u_{j} a_{I}$, where we denote $a(1)=\sum a_{I} e_{I}$ (1 is the multi-index, $\left\{e_{i}\right\}$ is the basis in $\left.F_{0}^{\prime}\right)$.

Hence from the previous diagram we see that

$$
\Delta_{X}=\sum(-1)^{j} u_{j} y_{j}
$$

This is the canonical expression for the discriminant in terms of the entries of $y$, and in terms of canonical factors of the minors of $d_{1}^{\prime}$. In this way the discriminant itself is explicitly calculated in terms of the complex $\mathbb{F}$.

\section{THE $G$-DISCRIMINANTS}

2.1. Let us consider a semisimple group $G$, and let $V=V_{\lambda}$ be the irreducible representation of highest weight $\lambda$, and $X$-the projectivisation of the orbit of the highest weight vector $v_{\lambda}$. The variety $X$ is the homogeneous space $G / P$ for the appropriate parabolic $P$, embedded by a complete linear system on $G / P$ corresponding to the line bundle $\mathscr{L}(\lambda)$ associated to the weight $\lambda$. If $\lambda=\sum \lambda_{i} \omega_{i}$ where $\omega_{i}$ are the fundamental weights, then $P$ is the parabolic corresponding to the subset $\left\{\alpha_{i} \mid \lambda_{i}>0\right\}$ of the basis $\left\{\alpha_{i}\right\}$ of the root system of $G$.

2.2. Examples. 1. The classical discriminant. Let us take $G=G L(n)$ to be the group of linear automorphisms of the $n$-dimensional space $F$. Let $\lambda=(m, 0, \ldots, 0)$. Then the corresponding representation $V_{\lambda}$ equals to the set $S_{m} F$ of symmetric tensors of rank $m$ on $F$. This space can be identified with the set of homogeneous polynomials of degree $m$ in $n$ variables. It follows 
directly from the definitions that the discriminant variety $X^{\vee}$ is the set of polynomials defining a singular hypersurface in $\mathbb{P}^{n-1}$. It follows that the variety $X^{\vee}$ has always codimensiom 1 and the discriminant $\Delta_{X}$ is in this case the classical discriminant of a homogeneous polynomial. The space $G / P$ in this case is just the projective space $\mathbb{P}(F)$. Let $0 \rightarrow \mathscr{R} \rightarrow F \rightarrow \mathscr{Q} \rightarrow 0$ be the tautological sequence on $\mathbb{P}(F)(\operatorname{dim} \mathscr{R}=1, \operatorname{dim} \mathscr{Q}=m-1)$. The bundle $\xi$ can be identified with $\mathscr{R}^{m-1} \otimes F$.

2. The hyperdeterminants. Let us consider $r$ vector spaces $F_{1}, \ldots, F_{r}$ of dimensions $m_{1}, \ldots, m_{r}$ respectively. Let us consider the group $G=G L\left(F_{1}\right) \times$ $\cdots \times G L\left(F_{r}\right)$ and let $V=F_{1} \otimes \cdots \otimes F_{r}$. The space $V$ can be identified with the set of $r$-dimensional matrices of the format $m_{1} \times \cdots \times m_{r}$. We will usually assume that $m_{1} \geq m_{2} \geq \cdots \geq m_{r}$. In this case the variety $Y$ is the orbit of the decomposable tensors. First we use our technique to calculate the codimension of the variety $X^{\vee}$.

Proposition [G-K-Z, K-M]. The codimension of the variety $X^{\vee}$ is equal to $\max \left(1, m_{1}-m_{2}-\cdots-m_{r}+r-1\right)$.

Proof. We will use the Theorem 1.7. In order to do this we have to identify the space $G / P$ and the bundle $\xi$. The space $G / P$ is the product of the projective spaces $\mathbb{P}\left(F_{1}\right) \times \cdots \times \mathbb{P}\left(F_{r}\right)$. Let $0 \rightarrow \mathscr{R}_{j} \rightarrow F_{j} \rightarrow \mathscr{Q}_{j} \rightarrow 0$ be the tautological sequence on $\mathbb{P}\left(F_{j}\right)$. Then the bundle $\xi$ is the sum (not direct!) of the subbundles $\mathscr{R}_{1} \otimes \cdots \otimes \mathscr{R}_{j-1} \otimes F_{j} \otimes \mathscr{R}_{j+1} \otimes \cdots \otimes \mathscr{R}_{r} \quad(1 \leq j \leq r)$ in $F_{1} \otimes \cdots \otimes F_{r}$.

The bundle $\xi$ is the extension of the direct sum $\xi^{\prime}=\bigoplus_{1 \leq j \leq r} \mathscr{R}_{1} \otimes \cdots \otimes \mathscr{R}_{j-1} \otimes$ $\mathscr{Q}_{j} \otimes \mathscr{R}_{j+1} \otimes \cdots \otimes \mathscr{R}_{r}$ by the line bundle $\mathscr{M}=\mathscr{R}_{1} \otimes \cdots \otimes \mathscr{R}_{j-1} \otimes \mathscr{R}_{j} \otimes \mathscr{R}_{j+1} \otimes \cdots \otimes \mathscr{R}_{r}$. The bundle $\Lambda^{j} \xi$ becomes the extension

$$
0 \rightarrow \bigwedge^{j} \xi^{\prime} \rightarrow \bigwedge^{j} \xi \rightarrow \bigwedge^{j-1} \xi \otimes \mathscr{M} \rightarrow 0 .
$$

The bundle $\Lambda^{j} \xi^{\prime}$ is the direct sum of the bundles

$$
\bigwedge^{a_{1}} \mathscr{Q}_{1} \otimes \mathscr{R}_{1}^{a_{2}+\cdots+a_{r}} \otimes \bigwedge^{a_{2}} \mathscr{Q}_{2} \otimes \mathscr{R}_{2}^{a_{1}+a_{3}+\cdots+a_{r}} \otimes \cdots \otimes \bigwedge^{a_{r}} \mathscr{Q}_{r} \otimes \mathscr{R}_{r}^{a_{1}+\cdots+a_{r-1}}
$$

(which we denote by $\left.\mathscr{V}\left(a_{1}, \ldots, a_{r}\right)\right)$ where we sum over all $r$-tuples $\left(a_{1}, \ldots\right.$, $\left.a_{r}\right)$ where $a_{1}+\cdots+a_{r}=j$. The bundle $\Lambda^{j-1} \xi \otimes \mathscr{M}$ is the direct sum of the bundles $\mathscr{V}\left(a_{1}, \ldots, a_{r}\right) \otimes \mathscr{M}$ where we sum over all $r$-tuples $\left(a_{1}, \ldots, a_{r}\right)$ where $a_{1}+\cdots+a_{r}=j-1$. The cohomology of each bundle $\mathscr{V}\left(a_{1}, \ldots, a_{r}\right)$ can be calculated by Bott's theorem. In fact we have to apply the Bott's theorem to the weights $\left(1^{a_{i}}, 0^{m_{i}-a_{i}-1}, a_{1}+\cdots+a_{i-1}+a_{i+1}+\cdots+a_{r}\right)$. The cohomology of the bundle $\mathscr{V}\left(a_{1}, \ldots, a_{r}\right) \otimes \mathscr{M}$ can be calculated in the same way by applying the Bott's theorem to the weight $\left(1^{a_{i}}, 0^{m_{i}-a_{i}-1}, a_{1}+\cdots+a_{i-1}+a_{i+1}+\cdots+a_{r}+1\right)$.

In calculation of the cohomology of $\Lambda^{j} \xi$ we have to take into account the cancellations occurring in the long homology sequence. They can be described as follows. Let us consider the bundle $\mathscr{V}\left(a_{1}, \ldots, a_{r}\right)$ such that the weight $\left(1^{a_{i}}, 0^{m_{i}-a_{i}-1}, a_{1}+\cdots+a_{i-1}+a_{i+1}+\cdots+a_{r}\right)$ is such that after adding $\rho$ (the half of sum of positive roots) and making the exchanges, the last number goes to the place $a_{i}+1$. Then the cohomology of such $\mathscr{V}\left(a_{1}, \ldots, a_{r}\right)$ (if nonzero) will cancel out with the cohomology of $\mathscr{V}\left(a_{1}, \ldots, a_{i}-1, \ldots, a_{r}\right) \otimes \mathscr{M}$ in the long cohomology sequence. This means that the cohomology of $\Lambda^{j} \xi$ is the direct sum of cohomologies of $\mathscr{V}\left(a_{1}, \ldots, a_{r}\right)$ such that for each weight 
$\left(1^{a_{i}}, 0^{m_{i}-a_{i}-1}, a_{1}+\cdots+a_{i-1}+a_{i+1}+\cdots+a_{r}\right)$ after applying the exchanges the last number either stays in place or moves to the first place, and of cohomologies of $\mathscr{V}\left(a_{1}, \ldots, a_{r}\right) \otimes \mathscr{M}$ such that for each weight $\left(1^{a_{i}}, 0^{m_{i}-a_{i}-1}, a_{1}+\cdots+a_{i-1}+\right.$ $\left.a_{i+1}+\cdots+a_{r}+1\right)$ the last number either stays in place or moves to the first place, but there it is not equal to 1 . Applying this principle, it is not hard to calculate $\max \left\{i \mid F_{i} \neq 0\right\}$.

When $m_{1}<m_{2}+\cdots+m_{r}-r+2$ then the cohomology of the top power of $\xi$ occurs in $F_{i}$ with $i=m_{1}+\cdots+m_{r}-r+1-\left(m_{1}-1\right)-\cdots-\left(m_{r}-1\right)=1$. It is easy to see in this case that all the terms $F_{i}$ with $i \geq 2$ have to be 0 . Let us assume that $m_{1} \geq m_{2}+\cdots+m_{r}-r+2$. Then all the pieces $\mathscr{V}\left(a_{1}, \ldots, a_{r}\right)$ and $\mathscr{V}\left(a_{1}, \ldots, a_{r}\right) \otimes \mathscr{M}$ contributing to the cohomology of $\Lambda^{j} \xi$ have the property that no exchanges occur after applying Bott's theorem to the weight corresponding to the first projective space.

This means that the only terms $\mathscr{V}\left(a_{1}, \ldots, a_{r}\right)$ and $\mathscr{V}\left(a_{1}, \ldots, a_{r}\right) \otimes \mathscr{M}$ contributing to the coholomogy of $\Lambda^{j} \xi$ are those with $a_{2}=\cdots=a_{r}=0$. The term in the highest $F_{i}$ is the term $\mathscr{V}\left(a_{1}, \ldots, a_{r}\right) \otimes \mathscr{M}$ where $a_{1}=m_{1}-1$, $a_{2}=\cdots=a_{r}=0$. It occurs with $i=m_{1}-\left(m_{2}-1\right)-\left(m_{3}-1\right)-\cdots-\left(m_{r}-1\right)=$ $m_{1}-m_{2}-\cdots-m_{r}+r-1$. This proves the proposition.

3. Another interesting case is the discriminant of Plücker embedding. Here we take $G=G L(F)$ and $\lambda=\left(1^{m}, 0^{n-m}\right)$. In this case $V=\Lambda^{m} F$. The variety $X^{\vee}$ is the dual variety of the grassmannian of $m$-planes in $n$ dimensional space.

2.3. Let us use the notation of 2.1. Let $\lambda^{\vee}$ be the lowest weight in $V_{\lambda}$. The bundle $\mathscr{S}$ (when pulled back to $G / B$ ) has a natural filtration whose associated graded object is the direct sum of line bundles $\mathscr{L}\left(\lambda^{\vee}+\alpha\right) \quad(\alpha>0,-\alpha$ is not in $\wp=\operatorname{Lie}(P))$ and $\mathscr{L}\left(\lambda^{\vee}\right)$. The number $e(\mathbb{F})$ can be calculated using this filtration.

\subsection{Proposition. In the above case}

$$
e(\mathbb{F})=\sum_{i, j \geq 0}(-1)^{i+1}(i+j) \operatorname{dim} H^{j}\left(G / B, \bigwedge^{i+j} \xi^{\prime}\right),
$$

where $\xi^{\prime}=\bigoplus_{\alpha>0,-\alpha \notin \emptyset} \mathscr{L}\left(\lambda^{\vee}+\alpha\right) \oplus \mathscr{L}\left(\lambda^{\vee}\right)$.

We can use Bott's theorem and Weyl's character formula to calculate the dimensions of cohomology groups $H^{j}\left(G / B, \Lambda^{i+j} \xi^{\prime}\right)$. This gives

$$
e(\mathbb{F})=\sum_{i, l \geq 0}(-1)^{i+1}(l) \sum \operatorname{ch}\left((l) \lambda^{\vee}+\alpha_{1}+\cdots+\alpha_{l}\right)
$$

where we sum over all $l$-tuples of different positive roots not in $\wp$, and $\operatorname{ch}(\mu)=$ $\prod_{\alpha>0}(\mu+\rho, \alpha) /(\rho, \alpha)$.

2.5. Remark. We can apply the technique of 2.4 to the twisted complex $\mathbb{F}(\mathscr{L})$ for any line bundle $\mathscr{L}$ on $X$. If $\mathscr{L}=\mathscr{L}(\beta)$ for some weight $\beta$, then we get

$$
e(\mathbb{F}(\mathscr{L}(\beta)))=\sum_{i, j \geq 0}(-1)^{i+1}(i+j) \operatorname{dim} H^{j}\left(G / B, \mathscr{L}(\beta) \otimes \bigwedge^{i+j} \xi^{\prime}\right),
$$

where $\xi^{\prime}=\bigoplus_{\alpha>0,-\alpha \text { not in } \wp} \mathscr{L}\left(\lambda^{\vee}+\alpha\right) \oplus \mathscr{L}\left(\lambda^{\vee}\right)$. Using Bott's theorem again, we get

$$
e(\mathbb{F}(\mathscr{L}(\beta)))=\sum_{i, l \geq 0}(-1)^{i+1}(l) \sum \operatorname{ch}\left(\beta+(l) \lambda^{\vee}+\alpha_{1}+\cdots+\alpha_{l}\right)
$$


where we sum over all $l$-tuples of different positive roots not in $\wp$, and $\operatorname{ch}(\mu)=$ $\prod_{\alpha>0}(\mu+\rho, \alpha) /(\rho, \alpha)$.

2.6. The remainder of this paper is devoted to the determinantal expressions for the discriminants. Remark 1.9 provides the natural approach to this problem. Let us suppose that $\operatorname{Codim} X^{\vee}=1$ (we are in the case (1a)). Let us assume that we have found the line bundle $\mathscr{L}$ on $X$ such that the complex $\mathbb{F}(\mathscr{L})$ has only two nonzero terms. These terms have to be $F(\mathscr{L})_{1}$ and $F(\mathscr{L})_{0}$. Then the whole complex $\mathbb{F}(\mathscr{L})$ reduces to a matrix. Moreover, we know that the cokernel of this matrix has the support contained in the variety $Y^{\vee}$. It follows from 2.5 that the determinant of the matrix has to be equal to the discriminant. This leads to many interesting expressions.

\section{THE DETERMINANTAL EXPRESSIONS FOR THE CLASSICAL DISCRIMINANTS}

3.1. Let us consider the determinantal expressions in the case of the classical discriminant of a homogeneous polynomial of degree $m$ in $n$ variables. We use the notation of Example 2.2, 1. Since $\xi=\mathscr{R}^{m-1} \otimes F$, we see that $\Lambda^{j} \xi=$ $\mathscr{R}^{j(m-1)} \otimes \bigwedge^{j} F$. The cohomology groups of these bundles are easily calculated using Serre's Theorem.

We will write the bundle $\mathscr{O}(a)$ in terms of its weight, i.e.

$$
\mathscr{O}(a)=\mathscr{L}\left(a_{1}, \ldots, a_{n}\right)
$$

for $a_{1}=\cdots=a_{n-1}=a, a_{n}=0$. In what follows we assume that $m \geq 3$, because the discriminant of the quadratic form is well known to be its determinant. We will denote by $S_{\left(a_{1}, \ldots, a_{n}\right)} F$ the Schur functor corresponding to the partition $\left(a_{1}, \ldots, a_{n}\right)$. In $\S 3$, since we deal with the projective spaces, most of the Schur functors that appear will be the symmetric powers of $F$ or $F^{*}$ tensored with the tensor powers of $\bigwedge^{n} F$. Only in the $\S 3.6$ some "real" Schur functors will appear.

3.2. Let us first assume that $n=2$. Then for any $-2<a<2(m-1)$ the complex $\mathbb{F}(\mathscr{O}(a))$ has two nonzero terms, so we get the determinantal expression of the discriminant. The possibilities $a=-1, a=m-2, a=2 m-3$ were considered above.

For $a=-1$ we get the format

$$
\bigwedge^{2} F \otimes S_{(2 m-3,0)} F \rightarrow F \otimes S_{(m-2,0)} F
$$

which gives the classical Sylvester formula for the discriminant. For $0 \leq a \leq$ $m-3$ we get the format

$$
\bigwedge^{2} F \otimes S_{(2 m-3, a+1)} F \rightarrow F \otimes S_{(m-2, a+1)} F \oplus S_{(a, 0)} F .
$$

This matrix consists of the linear and quadratic block.

For $a=m-2$ we get the format

$$
\bigwedge^{2} F \otimes S_{(2 m-3, m-1)} F \rightarrow S_{(m-2,0)} F .
$$

In this matrix all entries are quadratic. This is the classical Bezout formula for the discriminant. 
For $m-1 \leq a \leq 2 m-4$ we get the format

$$
\bigwedge^{2} F \otimes S_{(2 m-3, a+1)} F \oplus F \otimes S_{(a, m-1)} F \rightarrow S_{(a, 0)} F
$$

which again consists of the linear and quadratic block. Finally for $m=2 m-3$ we get the format

$$
F \otimes S_{(2 m-3, m-1)} F \rightarrow S_{(2 m-3,0)} F .
$$

The matrices coming from $\mathscr{O}(a)$ and from $\mathscr{O}(2 m-4-a)$ are transpose to each other because of Serre duality.

3.3. Let us consider the case $n=3$. The bundle $\mathscr{O}(a)$ leads to the two term complex only for $m-3 \leq a \leq 2 m-3$. Indeed, if $m-3>a$ then the term coming from $\bigwedge^{1} \xi(F \otimes \overline{\mathscr{L}}(a, a, m-1))$ gives the nonzero term in $H^{2}$, and therefore in the $(-1)$ st term of the complex. If $a>2 m-3$ then the term coming from $\Lambda^{2} \xi$ gives a nonzero term in the 2nd term of the complex.

Let us consider the formats we get.

For $a=m-3$ we get the format

$\bigwedge^{3} F \otimes S_{(3 m-5, m-2, m-2)} F \rightarrow \bigwedge^{2} F \otimes S_{(2 m-4, m-2, m-2)} F \oplus S_{(m-3, m-3,0)} F$.

For $a=m-2$ we get the format

$\bigwedge^{3} F \otimes S_{(3 m-5, m-1, m-1)} F \rightarrow \bigwedge^{2} F \otimes S_{(2 m-4, m-1, m-1)} F \oplus S_{(m-2, m-2,0)} F$.

For $m-1 \leq a \leq 2 m-5$ we get the format

$$
\begin{gathered}
\bigwedge^{3} F \otimes S_{(3 m-5, a+1, a+1)} F \oplus \bigwedge^{2} F \otimes S_{(2 m-4, a+1, a+1)} F \\
\rightarrow F \otimes S_{(a, a, m-1)} F \oplus S_{(a, a, 0)} F .
\end{gathered}
$$

For $a=2 m-4$ we get the format

$\bigwedge^{3} F \otimes S_{(3 m-5,2 m-3,2 m-3)} F \rightarrow F \otimes S_{(2 m-4,2 m-4, m-1)} F \oplus S_{(2 m-4,2 m-4,0)} F$.

Finally for $a=2 m-3$ we get the format

$$
\bigwedge^{3} F \otimes S_{(3 m-5,2 m-2,2 m-2)} F \rightarrow F \otimes S_{(2 m-3,2 m-3, m-1)} F \oplus S_{(2 m-3,2 m-3,0)} F \text {. }
$$

3.4. Next let us consider the case $n=4$. Looking at the term coming from $\mathscr{O}(a) \otimes \bigwedge^{2} \xi$, we see that its cohomology has to be 0 . Otherwise we would get the term in either $H^{0}$ or $H^{3}$ and both of them would ensure that the complex $\mathbb{F}(\mathscr{O}(a))$ has at least three terms. This means that $a=2 m-5,2 m-4$ or $2 m-3$.

We get the following formats:

$$
\begin{aligned}
& \bigwedge^{4} F \otimes S_{(4 m-7, a+1, a+1, a+1)} F \oplus F \otimes S_{(a, a, a, m-1)} F \\
& \rightarrow \bigwedge^{3} F \otimes S_{(3 m-6, a+1, a+1, a+1)} F \oplus S_{(a, a, a, 0)} F
\end{aligned}
$$

(the exception is that if $m=3, a=2 m-5$, then $S_{(a, a, a, m-1)} F$ equals to 0 . Similarly when $m=3$ and $a=2 m-3$, then the term $S_{(3 m-6, a+1, a+1, a+1)} F$ equals to 0 ). 
3.5. Finally, let us consider $n \geq 5$. Again looking at the term coming from $\mathscr{O}(a) \otimes \Lambda^{2} \xi$ we see that its cohomology has to vanish, and therefore $2 m-n-1 \leq$ $a<2 m-2$. Looking at the $(n-2)$ nd term we see that for the same reason $(n-2)(m-1)-n+1 \leq a<(n-2)(m-1)$. These two conditions cannot be satisfied when $(n-2)(m-1)-n+1 \geq 2(m-1)$, i.e. when $(n-4)(m-1) \geq n-1$.

Since $m-1 \geq 2$, we have $(n-4)(m-1) \geq 2(n-4)$. This means that for $n \geq 7$ the above inequality is always satisfied and the complex $\mathbb{F}(\mathscr{O}(a))$ has always at least three terms. It remains to consider $n=5$ and $n=6$.

For $n=5$ the above inequality reads $3(m-1)-4 \geq 2(m-1)$, i.e. $m \geq 5$. This means the only determinantal expression can occur for $m=3$ and $m=4$.

Let $m=4$. Looking at the 2 nd and 3 rd term we see that the only possible $a=5$. For this $a$ we get the following format

$$
\begin{aligned}
\bigwedge^{5} F & \otimes S_{(11,6,6,6,6)} F \oplus F \otimes S_{(5,5,5,5,3)} F \\
\rightarrow & \bigwedge^{4} F \otimes S_{(8,6,6,6,6)} F \oplus S_{(5,5,5,5,0)} F .
\end{aligned}
$$

Let $m=3$. Looking at the 2 nd and 3 rd term we see that the only possible $a=2$ or $a=3$. For $a=2$ we get the format

$$
\begin{aligned}
\bigwedge^{5} F & \otimes S_{(6,3,3,3,3)} F \oplus F \otimes S_{(2,2,2,2,2)} F \\
\rightarrow & \bigwedge^{4} F \otimes S_{(4,3,3,3,3)} F \oplus S_{(2,2,2,2,0)} F .
\end{aligned}
$$

For $a=3$ we get the transpose format

$$
\begin{aligned}
\bigwedge^{5} F & \otimes S_{(6,4,4,4,4)} F \oplus F \otimes S_{(3,3,3,3,2)} F \\
\rightarrow & \bigwedge^{4} F \otimes S_{(4,4,4,4,4)} F \oplus S_{(3,3,3,3,0)} F .
\end{aligned}
$$

Next we consider the case $n=6$. The above inequality shows that the only possible $m$ is $m=3$. Looking at the 2 nd, the 3 rd and the 4 th term we see that the only possible $a=3$. We get the following format

$$
\begin{aligned}
& \bigwedge^{6} F \otimes S_{(7,4,4,4,4,4)} F \oplus F \otimes S_{(3,3,3,3,3,2)} F \\
& \rightarrow \bigwedge^{5} F \otimes S_{(5,4,4,4,4,4)} F \oplus S_{(3,3,3,3,3,0)} F .
\end{aligned}
$$

The reasoning gives all possible determinantal expressions of the discriminant that can be obtained from the complexes $\mathbb{F}(\mathscr{L})$. The more precise description of the matrices described above will be the subject of the separate paper.

3.6. We saw above that it is possible to get the determinantal expressions for the discriminant of the form of degree $d$ in $n$ variables only for very special values of $d, n$. Now we show that for every $d, n$ it is possible to find a linear $G L(n)$-invariant matrix whose determinant is the power of the discriminant.

The idea is to pull back our construction to the full flag variety $G / B$ of $F$. Notice that after doing that we cannot apply the Remark 2.5. Still, if we find the bundle $\mathscr{L}$ over $G / B$ for which $\mathbb{F}(\mathscr{L})$ has only two terms, we get the matrix such that the support of its cokernel is contained in $Y^{\vee}$. This allows us to conclude that the determinant of this matrix is equal to the power of the discriminant. 
The line bundles on $G / B$ correspond to weights. Let us denote the line bundle corresponding to the weight $\left(a_{1}, \ldots, a_{n}\right)$ by $\mathscr{L}\left(a_{1}, \ldots, a_{n}\right)$. Let $\pi: G / B$ $\rightarrow G / P$ denote the natural projection. If we choose $\left(a_{1}, \ldots, a_{n}\right)$ in such way that $a_{1} \geq \cdots \geq a_{n-1}, a_{n}=0$, and consider the module $\mathscr{M}=\mathscr{L}\left(a_{1}, \ldots, a_{n}\right) \otimes$ $\pi^{*}\left(\mathscr{O}_{Z}\right)$, its higher direct images can be calculated by first pushing to $G / P=$ $\mathbb{P}(F)$ and then by calculating cohomology. By projection formula and by Bott's theorem $\mathscr{R}^{i} \pi_{*}(\mathscr{M})=0$ for $i>0$ and $\mathscr{R}^{0} \pi_{*}(\mathscr{M})=S_{\left(a_{1}, \ldots, a_{n-1}\right)} \mathscr{Q} \otimes \mathscr{O}_{Z}$. This means that we calculate the resolution of the module of rank bigger than one. In fact in this case the power of the discriminant that we get is equal to the rank of $S_{\left(a_{1}, \ldots, a_{n-1}\right)} \mathscr{Q}$.

Let us calculate the cohomology directly over $G / B$. It follows from the projection formula that the cohomology of $\mathscr{L}\left(a_{1}, \ldots, a_{n}\right) \otimes \pi^{*}\left(\bigwedge^{j} \xi\right)$ equals to $\Lambda^{j} F$ tensored with the cohomology of $\mathscr{L}\left(a_{1}, \ldots, a_{n}+j(m-1)\right)$.

This means that the bundle $\mathscr{L}\left(a_{1}, \ldots, a_{n}\right)$ gives us a determinantal expression of the power of the discriminant if only two of the bundles

$$
\mathscr{L}\left(a_{1}, \ldots, a_{n}+j(m-1)\right)
$$

for $0 \leq j \leq n$ have nonzero cohomology.

Let us fix two numbers $b_{1}$ and $b_{2}, 0 \leq b_{1}<b_{2} \leq n$. For each such pair $\left(b_{1}, b_{2}\right)$ there is a unique weight $\left(a_{1}, \ldots, a_{n}\right)$ for which $a_{1} \geq \cdots \geq a_{n-1}$, $a_{n}=0$, and the cohomology of $\Lambda^{j} \xi$ is zero except for $j=b_{1}$ and $j=b_{2}$. We see it easily from Bott's theorem. This weight is equal to

$$
\begin{aligned}
& (n(m-2)+1,(n-1)(m-2)+1, \ldots, \\
& \quad\left(b_{2}+1\right)(m-2)+1,\left(b_{2}-1\right)(m-2), \ldots, \\
& \left.\quad\left(b_{1}+1\right)(m-2),\left(b_{1}-1\right)(m-2)-1, \ldots,(m-2)-1,-1,0\right) .
\end{aligned}
$$

Let us consider the contribution from the $b_{2}$ th term. It is equal to the cohomology of the line bundle corresponding to the weight

$$
\begin{aligned}
& (n(m-2)+1,(n-1)(m-2)+1, \ldots, \\
& \quad\left(b_{2}+1\right)(m-2)+1,\left(b_{2}-1\right)(m-2), \ldots, \\
& \left.\quad\left(b_{1}+1\right)(m-2),\left(b_{1}-1\right)(m-2)-1, \ldots,(m-2)-1,-1, b_{1}(m-1)\right)
\end{aligned}
$$

tensored with $\bigwedge^{b_{1}} F$. Applying Bott's theorem we see that the nonvanishing cohomology occurs in degree $b_{1}$ and it is the irreducible corresponding to the weight

$$
\begin{aligned}
\mu_{1}= & (n(m-2)+1,(n-1)(m-2)+1, \ldots, \\
& \left(b_{2}+1\right)(m-2)+1,\left(b_{2}-1\right)(m-2), \ldots, \\
& \left.\left(b_{1}+1\right)(m-2), b_{1}(m-2),\left(b_{1}-1\right)(m-2), \ldots,(m-2), 0\right) .
\end{aligned}
$$

Thus the total contribution of the term $b_{1}$ is $\Lambda^{b_{1}} F \otimes S_{\mu_{1}} F$ in homological degree $b_{1}$.

Similarly we calculate that the contribution of the term $b_{2}$ is equal $\Lambda^{b_{2}} F \otimes$ $S_{\mu_{2}} F$ in cohomological degree $b_{2}-1$, where $\mu_{2}$ denotes the weight

$$
\begin{aligned}
\mu_{2}= & (n(m-2)+1,(n-1)(m-2)+1, \ldots, \\
& \left(b_{2}+1\right)(m-2)+1, b_{2}(m-2)+1,\left(b_{2}-1\right)(m-2)+1, \ldots, \\
& \left.\left(b_{1}+1\right)(m-2)+1,\left(b_{1}-1\right)(m-2), \ldots,(m-2), 0\right) .
\end{aligned}
$$


Thus the whole complex reduces to one map of homogeneous degree $b_{2}-b_{1}$,

$$
\bigwedge^{b_{2}} F \otimes S_{\mu_{2}} F \rightarrow \bigwedge^{b_{1}} F \otimes S_{\mu_{1}} F .
$$

It is an amusing exercise to apply the Weyl dimension formula to check that the dimensions of both tensor products are the same. The calculation of this dimension tells us also which power of the discriminant is the determinant of our matrix.

Let us note that in general the pairs $\left(b_{1}, b_{2}\right)$ and $\left(n-b_{2}, n-b_{1}\right)$ lead to the same determinantal expression because of Serre's duality.

These expressions seem to be particularly interesting for $b_{2}=b_{1}+1$ because in this case the terms of our matrix are linear.

Let us look at some examples. The case $n=2$ was treated above, because in this case $G / B=G / P$.

Let us consider in more detail the case of ternary form. It is enough to analyse the expressions coming from following pairs $\left(b_{1}, b_{2}\right):(0,1),(0,2),(0,3)$, $(1,2)$. Here are the formats we get: $(0,1)$ : we get the linear map

$$
F \otimes S_{(3 m-5,2 m-3, m-1)} F \rightarrow S_{(3 m-5,2 m-3,0)} F .
$$

Its determinant is the $(m-1)$ st power of the discriminant. $(0,2)$ : we get the quadratic map

$$
\bigwedge^{2} F \otimes S_{(3 m-5,2 m-3, m-1)} F \rightarrow S_{(3 m-5, m-2,0)} F .
$$

Its determinant is the $2(m-1)$ st power of the discriminant. $(0,3)$ : we get the cubic map

$$
\bigwedge^{3} F \otimes S_{(3 m-5,2 m-3, m-1)} F \rightarrow S_{(2 m-4, m-2,0)} F .
$$

Again the determinant is the $(m-1)$ st power of the discriminant. $(1,2)$ : we get the linear map

$$
\bigwedge^{2} F \otimes S_{(3 m-5,2 m-3,0)} F \rightarrow F \otimes S_{(3 m-5, m-2,0)} F .
$$

The determinant is the $3(m-1)$ st power of the discriminant.

The construction described here does not give all choices of $\mathscr{L}\left(a_{1}, \ldots, a_{n}\right)$ giving some determinantal expression for the power of the discriminant. It could happen that the cohomology of more than two terms does not vanish, but all nonvanishing terms occur in the 0th and 1st term of the complex $F(\mathscr{L})$.

\section{THE COMPLEXES $\mathbb{F}(\mathscr{L})$ AND THE DETERMINANTAL EXPRESSIONS FOR HYPERDETERMINANTS}

4.1. In this section we investigate the determinantal expressions for hyperdeterminants. Since the full classification of the determinantal expressions similar to the one described in $\S 3$ is very lengthy, we limit ourselves to providing some general principles of the calculation and the most interesting examples.

For the sake of simplicity we will consider mainly 3 dimensional matrices. We will indicate possible generalisations to higher dimensional matrices.

Let us denote by $F, G, H$ the three vector spaces of dimensions $f, g, h$ respectively. We will assume that $f \geq g \geq h$. By Proposition 2.2 (example 2) we can also assume that $f \leq g+h-1$. 
4.2. The variety $X$ is equal to $\mathbb{P}(F) \times \mathbb{P}(G) \times \mathbb{P}(H)$, the line bundles $\mathscr{L}$ we consider are $\mathscr{O}(u, v, w)=\mathscr{O}(u) \otimes \mathscr{O}(v) \otimes \mathscr{O}(w)$ where $u, v, w \in \mathbb{Z}$. The complex $\mathbb{F}(\mathscr{L})$ (the push down of $\Lambda \cdot(\xi) \otimes \mathscr{L}$ ) is denoted by $\mathbb{F}(u, v, w)$ for $\mathscr{L}=\mathscr{O}(u, v, w)$.

Let $\alpha, \beta, \gamma$ be three partitions. In this section we write sometimes $(\alpha) \otimes$ $(\beta) \otimes(\gamma)$ instead of $S_{(\alpha)} F \otimes S_{(\beta)} G \otimes S_{(\gamma)} H$.

We recall that the bundle $\xi$ is the extension of the direct sum

$$
\xi^{\prime}=\mathscr{R}_{F} \otimes \mathscr{R}_{G} \otimes \mathscr{Q}_{H} \oplus \mathscr{R}_{F} \otimes \mathscr{Q}_{G} \otimes \mathscr{R}_{H} \oplus \mathscr{Q}_{F} \otimes \mathscr{R}_{G} \otimes \mathscr{R}_{H}
$$

by the line bundle $\mathscr{M}=\mathscr{R}_{F} \otimes \mathscr{R}_{G} \otimes \mathscr{R}_{H}$. Therefore (as in $\S 2.2$, Example 2) the exterior power $\Lambda^{j} \xi$ is the extension

$$
0 \rightarrow \bigwedge^{j} \xi^{\prime} \rightarrow \bigwedge^{j} \xi \rightarrow \bigwedge^{j-1} \xi^{\prime} \otimes \mathscr{M} \rightarrow 0 .
$$

Let us denote by $\mathscr{V}(a, b, c)$ the bundle

$$
\mathscr{V}(a, b, c)=\bigwedge^{a} \mathscr{Q}_{F} \otimes \mathscr{R}_{F}^{b+c} \otimes \bigwedge^{b} \mathscr{Q}_{G} \otimes \mathscr{R}_{G}^{a+c} \otimes \bigwedge^{c} \mathscr{Q}_{H} \otimes \mathscr{R}_{H}^{a+b} .
$$

Then $\Lambda^{j} \xi^{\prime}=\bigoplus_{a+b+c=j} \mathscr{V}(a, b, c)$ where $a, b, c$ satisfy additional conditions $0 \leq a \leq f-1,0 \leq b \leq g-1,0 \leq c \leq h-1$. Similarly $\wedge^{j} \xi^{\prime} \otimes \mathscr{M}=$ $\bigoplus_{a+b+c=j-1} \mathscr{V}(a, b, c) \otimes \mathscr{M}$ where again $a, b, c$ satisfy $0 \leq a \leq f-1$, $0 \leq b \leq g-1,0 \leq c \leq h-1$.

We can calculate the cohomology of each piece $\mathscr{V}(a, b, c) \otimes \mathscr{L}$ (resp. $\mathscr{V}(a, b, c) \otimes \mathscr{M} \otimes \mathscr{L})$ by Bott's theorem. We just have to apply it to the weight

$\left((u+1)^{a}, u^{f-1-a}, b+c\right) \otimes\left((v+1)^{b}, v^{g-1-b}, a+c\right) \otimes\left((w+1)^{c}, w^{h-1-c}, a+b\right)$

(resp.

$$
\begin{aligned}
\left((u+1)^{a}, u^{f-1-a}, b+c+1\right) & \otimes\left((v+1)^{b}, v^{g-1-b}, a+c+1\right) \\
& \left.\otimes\left((w+1)^{c}, w^{h-1-c}, a+b+1\right)\right) .
\end{aligned}
$$

Let us consider the summand $\mathscr{V}(a, b, c) \otimes \mathscr{L}$ which contributes to the cohomology of $\Lambda^{j} \xi^{\prime}$ and which has the property that in applying Bott's theorem to the weight $\left((u+1)^{a}, u^{f-1-a}, b+c\right)$ the number $b+c$ moves to the $a+1$ th place. Then we can consider the corresponding summand $\mathscr{V}(a-1, b, c) \otimes \mathscr{M} \otimes \mathscr{L}$ and we notice that the cohomology of this summand is the same as of the original one, but in one degree lower. In this case the connecting homomorphism from the cohomology of $\mathscr{V}(a-1, b, c) \otimes \mathscr{M} \otimes \mathscr{L}$ to the cohomology of $\mathscr{V}(a, b, c) \otimes \mathscr{L}$ is nonzero, because the corresponding "piece" of the bundle $\Lambda^{j} \xi \otimes \mathscr{L}$ is induced by the tautological extension. The same rule applies to the second and third coordinate. This rule allows to describe cancellations in cohomology induced by the long exact sequence corresponding to the extension $(*)$.

After performing the cancellations described above we see that the cohomology of $\Lambda^{j} \xi \otimes \mathscr{L}$ consists of:

(A) the cohomologies of all pieces $\mathscr{V}(a, b, c) \otimes \mathscr{L}$, for which in each of the three coordinates when performing the exchanges prescribed by Bott's theorem the last number either stays in place or moves all the way to the first place; 
(B) the cohomologies of all pieces $\mathscr{V}(a, b, c) \otimes \mathscr{M} \otimes \mathscr{L}$, for which in each of the three coordinates when performing the exchanges prescribed by Bott's theorem the last number either stays in place or moves all the way to the first place and there it is not equal to $u+1$ (resp. $v+1, w+1$ ).

This principle allows us to analyse completely the complexes $\mathbb{F}(u, v, w)$.

Using the Serre duality for $Z$ and for $X$ we see easily that pushing down the complex $\Lambda \cdot \xi \otimes \mathscr{L}$ gives the dual complex to the one we get by pushing down $\bigwedge \cdot \xi \otimes \mathscr{L}^{-1} \otimes \omega$. Since $\omega=\mathscr{O}(g+h-f-2, f+h-g-2, f+g-h-2)$ we see that $\mathbb{F}(u, v, w)$ is dual to $\mathbb{F}(g+h-f-2-u, f+h-g-2-v, f+g-h-2-w)$.

Let us divide the set of all triples $(u, v, w)$ into 8 parts according to its position with respect to $\omega$. We will associate to a given $(u, v, w)$ the triple of signs \pm according to whether a given coordinate is bigger or smaller than the half of the corresponding coordinate of $\omega$ (with the equality counted as $+)$. For example $(u, v, w)$ is of the type $(+,-,+)$ if $u \geq \frac{1}{2}(g+h-f-2)$, $v<\frac{1}{2}(f+h-g-2), w \geq \frac{1}{2}(f+g-h-2)$.

Because of duality it is enough to consider 4 cases:

1. $(u, v, w)$ is of the type $(+,+,+)$.

2. $(u, v, w)$ is of the type $(+,+,-)$.

3. $(u, v, w)$ is of the type $(+,-,+)$.

4. $(u, v, w)$ is of the type $(-,+,+)$.

4.3. Example. Let us first consider the boundary case, i.e. $f=g+h-1$. In this case there are several interesting determinantal expressions:

(a) $(u, v, w)=(-1,2 h-4, g-3)$. Then after performing the calculation indicated above we get the determinant of the format

$$
\begin{aligned}
& \left(1^{f-1}, 0\right) \otimes\left((2 h-2)^{g}\right) \otimes\left(2 g-2,(g-1)^{h-1}\right)=\bigwedge^{f-1} F \otimes S_{g-1} H \\
& \uparrow \\
& \left(1^{f}\right) \otimes\left(2 h-1,(2 h-2)^{g-1}\right) \otimes\left(2 g-1,(g-1)^{h-1}\right)=\bigwedge^{f} F \otimes G \otimes S_{g} H .
\end{aligned}
$$

One can immediately identify the map above as the only invariant linear map over $\operatorname{Sym}(F \otimes G \otimes H)$. This is the determinantal expression described by Gelfand, Kapranov and Zelevinski in [G-K-Z3]. Of course the role of $G$ and $H$ can be reversed here.

(b) $(u, v, w)=(-1, h-2,2 g-3)$. This bundle comes to mind when we look at the bundles of type $(-,+,+)$. The conditions then read $u<-\frac{1}{2}, v \geq$ $\frac{1}{2}(2 h-3), w \geq \frac{1}{2}(2 g-3)$. If we pick $u$ and $v$ minimal possible, then we see that the only $w$ such that the bundle $(u, v, w)$ will lead to the determinantal expression is $w=2 g-3$. The calculation indicated above gives

$$
\begin{gathered}
\left(0^{f}\right) \otimes\left((h-1)^{g}\right) \otimes\left((2 g-2)^{h-1}, g-1\right)=S_{g-1} H^{*} \\
\uparrow \\
\left(1^{f}\right) \otimes\left(2 h-1, h^{g-1}\right) \otimes\left((2 g-1)^{h}\right)=\bigwedge^{f} F \otimes S_{h-1} G .
\end{gathered}
$$

The map is homogeneous of degree $f$. It is very easy to describe. In fact there is only one (up to scalar) invariant map of degree $f$ from $\wedge^{f} F \otimes S_{h-1} G$ to $S_{g-1} H^{*}$. It follows from the fact that $\operatorname{Sym}_{f}(F \otimes G \otimes H)$ by Cauchy formula contains the representation $\bigwedge^{f} F \otimes S_{\left(h, 1^{-1}\right)} G \otimes S_{\left(g, 1^{h-1}\right)} H$ exactly once. 
This example is easy to generalize to higher dimensional matrices. If the dimensions of our vector spaces are $f_{1}, f_{2}, \ldots, f_{r},\left(f_{1}=f_{2}+\cdots+f_{r}-r+2\right)$ then the bundle we have to use is $\mathscr{L}=\mathscr{O}\left(u_{1}, \ldots, u_{r}\right)$ where $u_{1}=-1, u_{j}=$ $2 f_{2}+\cdots+2 f_{j-1}+f_{j+1}+\cdots+f_{r}-j-1$ for $2 \leq j \leq r$. The determinantal expression becomes

$$
\begin{gathered}
S_{f_{2}-1} F_{3}^{*} \otimes S_{f_{2}+f_{3}-2} F_{4}^{*} \otimes \cdots \otimes S_{f_{2}+\cdots+f_{r-1}-r+2} F_{r}^{*} \\
\uparrow \\
\bigwedge^{f_{1}} F_{1} \otimes \bigwedge^{f_{2}} F_{2} \otimes S_{f_{3}+\cdots+f_{r}-r+2} F_{2} \otimes \cdots \otimes \bigwedge^{f_{r-1}} F_{r-1} \otimes S_{f_{r}-1} F_{r-1} \otimes \bigwedge^{f_{r}} F_{r} .
\end{gathered}
$$

The map is of degree $f_{1}$ in the entries of the matrix.

4.4. Example. The subboundary case. Let us assume that $f=g+h-2$.

We take the approach similar to the example $1(b)$. Let us consider the bundles of the type $(-,+,+)$. The conditions for $u, v, w$ give $u<0, v \geq h-2$, $w \geq g-2$. Let us pick $u=-1, v=h-2, w=2 g-3$ (the choice of $w$ is the only one leading to the determinantal expression). We get the following format:

$$
\begin{gathered}
\left(0^{f}\right) \otimes\left((h-1)^{g-1}, h-2\right) \otimes\left((2 g-2)^{h-2}, 2 g-3, g-1\right) \\
\uparrow \\
\left(1,0^{f-1}\right) \otimes\left((h-1)^{g}\right) \otimes\left((2 g-2)^{h-2}, 2 g-3, g\right) \\
\left(1^{g}, 0^{f-g}\right) \otimes\left((h)^{g-1}, h-1\right) \otimes\left((2 g-2)^{h}\right) .
\end{gathered}
$$

The map from the first piece is linear and one can see immediately that it is uniquely defined (up to scalar). The map from the second piece is of degree $g$. It is also uniquely defined because the only representation from $\operatorname{Sym}_{g}(F \otimes G \otimes H)$ that can possibly be involved is $\bigwedge^{g} F \otimes S_{\left(2,1^{-2}\right)} G \otimes S_{(g-1,1)} H$ which occurs in $\operatorname{Sym}_{g}(F \otimes G \otimes H)$ once.

This example can also be generalized to higher dimensional subboundary case. If the dimensions of our vector spaces are $f_{1}, f_{2}, \ldots, f_{r},\left(f_{1}=f_{2}+\cdots+\right.$ $\left.f_{r}-r+2\right)$ then the bundle we have to use is $\mathscr{L}=\mathscr{O}\left(u_{1}, \ldots, u_{r}\right)$ where $u_{1}=-1$, $u_{j}=2 f_{2}+\cdots+2 f_{j-1}+f_{j+1}+\cdots+f_{r}-j-1$ for $2 \leq j \leq r$. The corresponding complex contains in the 0th term $r-2$ representations, all in one degree $f_{1}-1$. The 1st term contains $r-2$ representations in degree $f_{1}$ and additional $r-2$ terms in degrees $f_{1}+f_{2}-1, f_{1}+f_{2}+f_{3}-2, \ldots, f_{1}+\cdots+f_{r-1}-r+1$.

4.5. Example. Here we give the example of 3 dimensional matrix for which no choice of $(u, v, w)$ gives a complex with two terms. In order to do this, let us consider the case of cubic matrices, i.e. $f=g=h$.

It is enough to consider the cases when $(u, v, w)$ is of type $(+,+,+)$ and of type $(+,+,-)$.

Case 1. The condition means that $u \geq \frac{1}{2}(f-2), v \geq \frac{1}{2}(f-2), w \geq \frac{1}{2}(f-2)$.

It is clear from the condition that $u, v, w$ are nonnegative. Let us look at the terms of type (A) in $H^{0}\left(\bigwedge^{2} \xi \otimes \mathscr{O}(u, v, w)\right)$. It is clear that if two of the numbers $u, v, w$ are $\geq 2$ or if all of them are $\geq 1$, but one $\geq 2$, then $H^{0}\left(\bigwedge^{2} \xi \otimes \mathscr{O}(u, v, w)\right) \neq 0$ and $\mathbb{F}(\mathscr{O}(u, v, w))$ has at least 3 terms. This settles the cases in which $f \geq 6$ (in the sense that all complexes $\mathbb{F}(\mathscr{O}(u, v, w)$ ) of type $(+,+,+)$ have at least 3 terms). 
Case 2. Let us assume that $(u, v, w)$ is of the type $(+,+,-)$. This means $u \geq \frac{1}{2}(f-2), v \geq \frac{1}{2}(f-2), w<\frac{1}{2}(f-2)$. In fact, by the same argument as in Case 1 , we can assume that $w<0$. Let us consider the case (A).

The bundle $\mathscr{V}(a, b, c)$ corresponds to the weight $\left((u+1)^{a}, u^{f-1-a}, b+\right.$ $c) \otimes\left((v+1)^{b}, v^{f-1-b}, a+c\right) \otimes\left(w^{f-1}, a+b\right)$. Let us consider $a=b=f-2$, $c=f-1$. If $u, v \leq f-3$, then $u+1+f-1 \leq 2 f-2<2 f-1$, and $v+1+f-1 \leq 2 f-2<2 f-1$. This shows that $\mathscr{V}(a, b, c)$ gives nonzero contribution to $H(3 f-3,3 f-5)$. This means that the complex $\mathbb{F}(\mathscr{O}(u, v, w))$ has at least 3 terms. Thus we can assume that $u \geq f-2$ (or $v \geq f-2$ ). To fix the notation let us assume that $u \geq f-2$. Let us recall that $v \geq \frac{1}{2}(f-2)$, $w<0$.

We consider $\mathscr{V}(a, b, 0)$ with $a=f-2, b=v$. Let us assume that $f \geq 8$. This means that $a+b \geq f-2+\frac{1}{2}(f-2) \geq f-2+3=f+1$. But $w+f-1<f+1$, so $\mathscr{V}(a, b, 0)$ defines a nonzero element of $H(f-1, f+1)$. This shows that for $f \geq 8$ there is no $(u, v, w)$ such that the complex $\mathbb{F}(\mathscr{O}(u, v, w))$ has two terms.

4.6. Example. We consider here the case $u=v=w=0$. We give the complete descriptions of the terms in the complex $\mathbb{F}$. We will denote our matrix by $A=\left(a_{i j k}\right)$ where $1 \leq i \leq f, 1 \leq j \leq g, 1 \leq k \leq h$. Sometimes we will treat this matrix as a trilinear form $\sum a_{i j k} x_{i} y_{j} z_{k}$ where $x_{i}(1 \leq i \leq f), y_{j}$ $(1 \leq j \leq g), z_{k}(1 \leq k \leq h)$ are three sets of variables. Thus we can talk about the partial derivatives $\partial A / \partial x_{i}, \partial A / \partial y_{j}, \partial A / \partial z_{k}$. The trilinear form $A$ and its partial derivatives are the elements of the polynomial ring $R=\mathbb{C}[x, y, z]=$ $\operatorname{Sym}(F \oplus G \oplus H)$. We will denote by $S_{\left(a_{1}, \ldots, a_{f}\right)} F$ the irreducible representation of $G L(F)$ corresponding to highest weight $\left(a_{1}, \ldots, a_{f}\right)$. Similarly for $G$ and $H$. The complex $\mathbb{F}$ defined above consists in this case of three parts:

(1) The trivial representation $S_{(0, \ldots, 0)} F \otimes S_{(0, \ldots, 0)} G \otimes S_{(0, \ldots, 0)} H$ in degree 0 (corresponding to $H^{0}\left(\bigwedge^{0}(\xi)\right)$.

(2) The complex

$$
\begin{gathered}
\bigwedge^{f} F \otimes S_{(f-g+1,1, \ldots, 1)} G \otimes S_{(f-h+1,1, \ldots, 1)} H \\
\downarrow \\
\bigwedge^{f-1} F \otimes S_{(f-g, 1, \ldots, 1)} G \otimes S_{(f-h, 1, \ldots, 1)} H \\
\downarrow \\
\ldots \ldots \ldots \ldots \ldots \ldots \ldots \ldots \ldots \ldots \ldots \ldots \\
\downarrow \\
\bigwedge^{g} F \otimes S_{(1,1, \ldots, 1)} G \otimes S_{(g-h+1,1, \ldots, 1)} H .
\end{gathered}
$$

The term $\bigwedge^{f} F \otimes S_{(f-g+1,1, \ldots, 1)} G \otimes S_{(f-h+1,1, \ldots, 1)} H$ appears in degree $f$ $g-h+2$. It is an element of $H^{g+h-2}\left(\bigwedge^{f} \xi\right)$. The differentials are linear in the entries of $A$ and one can see easily that this complex can be identified with the homogeneous component of the Koszul complex on $\partial A / \partial x_{i}$ over the polynomial ring $\operatorname{Sym}(G \oplus H)$. This part of the complex becomes a little bigger when $f=g$. In this case it contains additionally the terms $S_{(1, \ldots, 1)} F \otimes$ $S_{(1, \ldots, 1)} G \otimes S_{\left(b-h+1,2^{f-b}, 1, \ldots, 1\right)} H$ for $\max (h+1, f-h+1) \leq b \leq f-1$, in degrees 
$f-(f-1+f-b-1+h-1)=b-f-h+3$. For $f=g=h$ these additional terms become $f-1$ copies of $S_{(1, \ldots, 1)} F \otimes S_{(1, \ldots, 1)} G \otimes S_{(1, \ldots, 1)} H$ in degree $f-(f-1+f-2)=3-f$. In this case one of these terms cancels out with the term $S_{(1, \ldots, 1)} F \otimes S_{(1, \ldots, 1)} G \otimes S_{(1, \ldots, 1)} H$ in degree $2-f$ of the complex above, so the whole second part consists of $f-2$ copies of $S_{(1, \ldots, 1)} F \otimes S_{(1, \ldots, 1)} G \otimes S_{(1, \ldots, 1)} H$ in degree $f-(f-1+f-2)=3-f$.

(3) The third part appears only in the interior case. It consists of all terms in top cohomology $\left(H^{f+g+h-3}\right)$. It starts in degree 1 as follows:

$$
\begin{gathered}
S_{(g+h-f, 2, \ldots, 2)} F \otimes S_{(f+h-g, 2, \ldots, 2)} G \otimes S_{(f+g-h, 2, \ldots, 2)} H \\
\downarrow \\
S_{(g+h-f-1,2, \ldots, 2)} F \otimes S_{(f+h-g-1,2, \ldots, 2)} G \otimes S_{(f+g-h-1,2, \ldots, 2)} H \\
S_{(g+h-f-1,2, \ldots, 2)} F \otimes S_{(f+h-g-1,2, \ldots, 2)} G \otimes S_{(f+g-h, 2, \ldots, 2,1)} H \\
S_{(g+h-f-1,2, \ldots, 2)} F \otimes S_{(f+h-g, 2, \ldots, 2,1)} G \otimes S_{(f+g-h-1,2, \ldots, 2)} H \\
S_{(g+h-f, 2, \ldots, 2,1)} F \otimes S_{(f+h-g-1,2, \ldots, 2)} G \otimes S_{(f+g-h-1,2, \ldots, 2)} H \\
\downarrow
\end{gathered}
$$

The differentials in this complex are linear in the entries of $A$. If one of the sequences is not a partition, then the corresponding term disappears from the complex. Let us interpret this complex in terms of Koszul complex on partial derivatives of $A$. In fact we will give such interpretation to the dual complex. We will interpret the terms as homogeneous components of certain modules over $R=\operatorname{Sym}(F \oplus G \oplus H)$. The terms

$$
S_{(g+h-f, 2, \ldots, 2)} F \otimes S_{(f+h-g, 2, \ldots, 2)} G \otimes S_{(f+g-h, 2, \ldots, 2)} H
$$

and

$$
S_{(g+h-f-1,2, \ldots, 2)} F \otimes S_{(f+h-g-1,2, \ldots, 2)} G \otimes S_{(f+g-h-1,2 \ldots, 2)} H
$$

are just the homogeneous components of the $R$ itself. The map will obviously be the multiplication by $A$. The other two terms are more interesting. Let us look at

$$
S_{(g+h-f, 2, \ldots, 2,1)} F \otimes S_{(f+h-g-1,2, \ldots, 2)} G \otimes S_{(f+g-h-1,2, \ldots, 2)} H .
$$

It is a homogeneous component of the following (nonfree) module $M$ over $R$. $M=\sum_{a, b, c \geq 0} S_{(a, 0, \ldots, 0,-1)} F \otimes S_{(b, 0, \ldots, 0)} G \otimes S_{(c, 0, \ldots, 0)} H$. This module has the following free resolution over $R$ :

$$
0 \rightarrow R \rightarrow F^{*} \rightarrow M \rightarrow 0
$$

with the map of degree $(1,0,0)$ in $x, y, z$.

Each term of our complex has similar interpretation. The whole complex becomes isomorphic to the dual of the $E^{1}$-term of a homogeneous component of the following double complex over $R$.

$0 \longleftarrow R \longleftarrow \bigwedge^{1}\left(F^{*} \oplus G^{*} \oplus H^{*} \oplus R\right) \longleftarrow \bigwedge^{2}\left(F^{*} \oplus G^{*} \oplus H^{*} \oplus R\right) \longleftarrow \cdots$

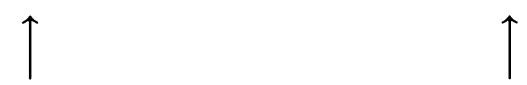

$R \oplus R \oplus R$

$\bigwedge^{1}\left(F^{*} \oplus G^{*} \oplus H^{*} \oplus R\right)^{\oplus 3}$ 
The horizontal differentials come from Koszul complex on $A, \partial A / \partial x_{i}, \partial A / \partial z_{k}$. The vertical maps are induced by the Euler relations:

$$
\sum x_{i} \partial A / \partial x_{i}=A, \quad \sum y_{j} \partial A / \partial y_{j}=A, \quad \sum z_{k} \partial A / \partial z_{k}=A .
$$

Other homogeneous components of this complex are parts of the twisted complexes $\mathbb{F}(\mathscr{L})$ for the appropriate line bundles $\mathscr{L}$ over $X$.

All of this allows us to identify the homology of the Koszul-Euler complex above (adjusted by parts (1), (2)) with the stalks of $\mathscr{R}^{j} q_{*}\left(\mathscr{O}_{Z}(\mathscr{L})\right.$ ) over a particular $A$. Let us look at the following special case. Let us assume that $f<g+h-2$ (so the complex 2) occurs in negative degrees).

Let $J(A)$ be the ideal generated by all partial derivatives of $A$. Then our complex tells us that hyperdiscriminant of $A \neq 0$ if and only if the homogeneous component of $R / J(A)$ in multidegree $(g+h-f-2, f+h-g-2, f+$ $g-h-2)$ is 1-dimensional (otherwise this component has bigger dimension).

This is an analogue of the well-known statement for the classical discriminant. There we know that $\operatorname{disc}(F) \neq 0$ if and only if the partials $F_{x_{1}}, \ldots, F_{x_{n}}$ form a regular sequence, if and only if the homogeneous component of $R / J(F)$ in degree $n(d-2)$ has dimension 1 .

It would be very interesting to generalize this approach to the case of arbitrary $G$-discriminant. In fact the complex $\mathbb{F}$ tells us what should be the proper definitions of partial derivatives. It seems that there almost always is a "top" 1 dimensional component. It would be also interesting to work out in more detail what is the Koszul cohomology of $J(A)$ for $A$ with nonzero discriminant.

4.7. Example. Here we consider the case when the codimension of the variety $X^{\vee}$ is bigger than 1. Then it is interesting to consider the defining ideal of the variety $X^{\vee}$. It turns out that in some small cases the technique of this paper allows to calculate the chain of syzygies of this ideal. The complex that one gets is the right analogue of the Eagon-Northcott complex for higher dimensional matrices.

We give examples of calculating this complex. One should observe that the set-theoretic equations of the hyperdeterminant variety are given by the rank conditions for the complex $\mathbb{F}$ described above, i.e. by the minors of given size of the matrix $F_{1} \rightarrow F_{0}$ described explicitly above.

(a) Let us first consider the case of $m \times 2 \times 2$ matrix $(m \geq 4)$. The complex $\mathbb{F}$ according to the above description looks as follows:

$$
\begin{aligned}
& \left(0^{m}\right) \otimes(0,0) \otimes(0,0) \oplus\left(1^{2}, 0^{m-2}\right) \otimes(1,1) \otimes(1,1) \\
& \uparrow \\
& \left(1^{3}, 0^{m-3}\right) \otimes(2,1) \otimes(2,1) \\
& \uparrow \\
& \left(1^{m}\right) \otimes(m-1,1) \otimes(m-1,1)
\end{aligned}
$$

Since no terms occur in negative homological degree, the complex $\mathbb{F}$ is a resolution of the normalisation of the coordinate ring of the hyperdeterminant variety. In order to get the syzygies of the coordinate ring itself, we have to 
resolve the cokernel of the map $\left(1^{3}, 0^{m-3}\right) \otimes(2,1) \otimes(2,1) \rightarrow\left(1^{2}, 0^{m-2}\right) \otimes$ $(1,1) \otimes(1,1)$. For this we use the following well-known construction. Let us consider the grassmannian of $m$-2-planes in $F$. Let $0 \rightarrow \mathscr{R} \rightarrow F \rightarrow \mathscr{Q} \rightarrow 0$ be the tautological sequence over this grassmannian $(\operatorname{dim} \mathscr{R}=m-2, \operatorname{dim} \mathscr{Q}=2)$. We consider the twisted Koszul complex $\Lambda^{2} \mathscr{Q} \otimes \Lambda(\mathscr{R} \otimes G \otimes H)$. We push down this complex to get the complex

$$
\begin{aligned}
\mathbb{C}: & \bigwedge^{2} F \leftarrow \bigwedge^{3} F \otimes U \leftarrow \bigwedge^{4} F \otimes S_{2} U \oplus S_{222} F \otimes \bigwedge^{4} U \leftarrow \cdots \\
& \leftarrow \bigwedge^{j+2} F \otimes S_{j} U \oplus \bigoplus_{|\alpha|=j-2, \alpha_{1} \leq 2} S_{222, \alpha} F \otimes \bigwedge^{4, \alpha} U \leftarrow \cdots
\end{aligned}
$$

where we write $U$ to denote $G \otimes H$. The complex $\mathbb{C}$ is acyclic except in degree 0 , since the complex $\Lambda^{2} \mathscr{Q} \otimes \Lambda(\mathscr{R} \otimes G \otimes H)$ resolves the module $\Lambda^{2} \mathscr{Q} \otimes$ $\operatorname{Sym}(\mathscr{Q} \otimes G \otimes H)$ which has no higher direct images. Therefore $\mathbb{C} \otimes \Lambda^{2} G \otimes \Lambda^{2} H$ resolves the cokernel we are interested in. This means that there exists a chain map $\mathbb{F} \rightarrow \mathbb{C} \otimes \bigwedge^{2} G \otimes \bigwedge^{2} H$ which resolves the natural epimorphism on 0th homology. The cone of this map is not minimal. After cancelling the repeating terms we get the following resolution of the hyperdeterminant variety in this case:

$$
\begin{aligned}
& \left(0^{m}\right) \otimes(0,0) \otimes(0,0) \\
& \left(2^{3}, 0^{m-3}\right) \otimes(3,3) \otimes(3,3) \oplus\left(1^{4}, 0^{m-4}\right) \otimes(2,2) \otimes(2,2) \\
& \uparrow \\
& \uparrow \\
& \bigoplus_{|\gamma|=j+1, \gamma \neq(j+1)} \bigwedge^{j+3} F \otimes \bigwedge^{2} G \otimes \bigwedge^{2} H \otimes S_{\gamma} G \otimes S_{\gamma} H \\
& \oplus \\
& \bigoplus_{|\beta|=j-1, \beta_{1} \leq 2} S_{(222, \beta)} F \otimes S_{(33)} G \otimes S_{(33)} H \otimes \bigwedge^{\beta}(G \otimes H) \\
& \uparrow
\end{aligned}
$$

From this we can conclude that the homological dimension of the coordinate ring is equal to $2 m-5$, the last term in the complex being $S_{\left(2^{m}\right)} F \otimes S_{33} G \otimes$ $S_{33} H \otimes \bigwedge^{\left(2^{m-3}\right)}(G \otimes H)$. Since the codimension of the hyperdeterminant variety in this case is equal to $m-2$, the defining ideal is never perfect.

It is easy to identify the generators of hyperdeterminantal ideal. The representation $\left(1^{4}, 0^{m-4}\right) \otimes(2,2) \otimes(2,2)$ corresponds to the $4 \times 4$ minors of the matrix we get from $m \times 2 \times 2$ matrix when we write one $m \times 2$ slice under another. The representation $\left(2^{3}, 0^{m-3}\right) \otimes(3,3) \otimes(3,3)$ is the representation generated by the hyperdeterminants of $3 \times 2 \times 2$ submatrices of our matrix. All basis elements of this representation can be obtained from hyperdeterminants of $3 \times 2 \times 2$ submatrices by means of various polarisation operators. 
(b) Let us consider $m \times 3 \times 2$ matrices. In this case the complex $\mathbb{F}$ looks as follows:

$$
\begin{aligned}
& \left(0^{m}\right) \otimes(0,0,0) \otimes(0,0) \oplus\left(1^{3}, 0^{m-3}\right) \otimes(1,1,1) \otimes(2,1) \\
& \uparrow \\
& \left(1^{4}, 0^{m-4}\right) \otimes(2,1,1) \otimes(3,1) \\
& \uparrow \\
& \left(1^{m}\right) \otimes(m-2,1,1) \otimes(m-1,1)
\end{aligned}
$$

Let us consider for a while the $4 \times 3 \times 2$ matrix. The above complex reduces then to

$$
\begin{gathered}
\left(0^{4}\right) \otimes(0,0,0) \otimes(0,0) \oplus\left(1^{3}, 0\right) \otimes(1,1,1) \otimes(1,1) \\
\uparrow \\
\left(1^{4}\right) \otimes(2,1,1) \otimes(3,1)
\end{gathered}
$$

This complex resolves the normalisation $\bar{S}$ of the coordinate ring $S$ of the hyperdeterminantal variety. But the ideal of this variety is principal, generated by an element of degree 12 . This means that the cokernel $\bar{S} / S$ for $4 \times 3 \times 2$ matrix has the resolution $\mathbb{C}$ which looks as follows:

$$
\begin{gathered}
\left(1^{3}, 0\right) \otimes(1,1,1) \otimes(1,1) \\
\uparrow \\
\left(1^{4}\right) \otimes(2,1,1) \otimes(3,1) \\
\uparrow \\
\left(3^{4}\right) \otimes\left(4^{3}\right) \otimes(6,6)
\end{gathered}
$$

Now we come back to arbitrary $m \times 3 \times 2$ matrix. The complex $\mathbb{F}$ is the resolution of $\bar{S}$. In order to find the resolution of $\bar{S} / S$ we consider the grassmannian Grass $(m-4, F)$ with the tautological sequence $0 \rightarrow \mathscr{R} \rightarrow F \rightarrow \mathscr{Q} \rightarrow 0$ $(\operatorname{dim} \mathscr{R}=m-4, \operatorname{dim} \mathscr{Q}=4)$. Then we take the complex $\mathbb{C}$ above (in a relative situation, i.e. for $\mathscr{Q}$ instead of $F$ ) tensored with $\Lambda(\mathscr{R} \otimes G \otimes H)$. This complex resolves the module containing the bundles of the type $S_{\alpha} \mathscr{Q} \otimes S_{\beta} G \otimes S_{\gamma} H$, so the push-down of this complex is acyclic in degrees $>0$ by Bott's theorem. This push-down is the total complex of the following double complex:

$$
\begin{aligned}
& \wedge^{3} F \otimes \wedge^{3} G \otimes S_{21} H \quad \leftarrow \quad \wedge^{4} F \otimes S_{211} G \otimes S_{31} H \quad \leftarrow S_{\left(3^{4}\right)} F \otimes S_{\left(4^{3}\right)} G \\
& \Lambda^{5} F \otimes \Lambda^{3} G \otimes S_{21} H \otimes \Lambda^{2} U \leftarrow \Lambda^{5} F \otimes S_{211} G \otimes S_{31} H \otimes U \leftarrow S_{\left(3^{4}\right)} F \otimes S_{\left(4^{3}\right)} G \\
& \Lambda^{6} F \otimes \Lambda^{3} G \otimes S_{21} H \otimes \Lambda^{21} U \leftarrow \\
& \oplus S_{\left(2^{4}, 1\right)} A \otimes \Lambda^{3} G \otimes S_{21} H \otimes \Lambda^{6} U
\end{aligned}
$$

where $U$ denotes $G \otimes H$. The left map in the second row is not minimal, so cancellations occurs. After performing the cancellations we get the following 
minimal complex $\mathbb{L}$ :

$$
\begin{gathered}
\bigwedge^{3} F \otimes \bigwedge_{\uparrow}^{3} G \otimes S_{21} H \\
\bigwedge^{4} F \otimes S_{211} G \otimes S_{31} H \\
\uparrow \\
S_{\left(3^{4}\right)} F \otimes S_{\left(4^{3}\right)} G \otimes S_{(6,6)} H \oplus \\
\bigwedge^{5} F \otimes S_{311} G \otimes S_{41} H \oplus \\
\bigwedge^{6} F \otimes S_{222} G \otimes S_{33} H \\
\uparrow
\end{gathered}
$$

This means that this is a resolution of the cokernel $\bar{S} / S$. Taking a mapping cone of the map $\mathbb{F} \rightarrow \mathbb{L}$ covering the natural surjection we finally get the following minimal resolution of $S$ :

$$
\begin{gathered}
\left(0^{m}\right) \otimes(0,0,0) \otimes(0,0) \\
\uparrow \\
\left(3^{4}, 0^{m-4}\right) \otimes\left(4^{3}\right) \otimes(6,6) \oplus \\
\left(1^{6}\right) \otimes\left(2^{3}\right) \otimes(3,3) \\
\uparrow
\end{gathered}
$$

This shows that the hyperdeterminant ideal is generated by the polarisations of hyperdeterminants of $4 \times 3 \times 2$ submatrices and (for $\operatorname{dim} F \geq 6$ ) by the $6 \times 6$ minors of $m \times 6$ matrix obtained from $m \times 3 \times 2$ matrix by writing one $m \times 3$ slice under another. Of course for $\operatorname{dim} F=5$ these generators disappear.

These two cases are the basis for the following conjecture:

Conjecture. Let us consider $m \times n \times p$ matrices, $m \geq n+p$. Then the hyperdeterminantal ideal is generated by polarisations of hyperdeterminants of $(n+p-1) \times n \times p$ matrices and (for $m \geq n p)$ by $n p \times n p$ minors of the $m \times n p$ matrix obtained from the original matrix by writing $p \quad m \times n$ slices one under another.

\section{REFERENCES}

[B-E] D. A. Buchsbaum and D. Eisenbud, Some structure theorems for finite free resolutions, Adv. in Math. 12 (1974), 84-139.

[E-N] J. Eagon and D. G. Northcott, On the Buchsbaum-Eisenbud theory of free resolutions, J. Reine Angew. Math. 262/263 (1973), 205-219.

[G-K-Z1] I. M. Gelfand, M. Kapranov, and A. Zelevinski, Projective duality of varieties and hyperdeterminant, Dokl. Akad. Nauk SSSR 305 (1989), 1294-1298; Soviet Math. Dokl. 39 (1989), no. 2.

[G-K-Z2] _ , A-discriminants and the Cayley-Koszul complexes, Dokl. Akad. Nauk SSSR 307 (1989), 1307-1311. 
[G-K-Z3] _ General discriminants, List of results. Preprint, 1989.

[H] R. Hartshorne, Algebraic geometry, Springer-Verlag, New York, Heidelberg, and Berlin, 1977.

[Ho] A. Holme, The geometric and numerical properties of duality in projective algebraic geometry, Manuscripta Math. 61 (1988), 145-162.

[Ka] N. Katz, Pinceaux de Lefschetz: theoreme d'existence, SGA 7 11, Expose XVII, Lecture Notes in Math., vol. 340, Springer-Verlag, 1973, pp. 212-253.

[K] S. L. Kleiman, The enumerative theory of singularities, Real and Complex Singularities, Oslo, 1976, pp. 297-396.

[K-M] F. Knop and G. Mentzel, Duale Varietaten von Fahnenvarietaten, Comment. Math. Helv. 62 (1987), 38-61.

[KKMS] G. R. Kempf, F. Knudsen, D. Mumford, and B. Saint-Donat, Toroidal embeddings. I, Lecture Notes in Math., vol. 339, Springer-Verlag, 1973.

[S] G. Salmon, Lessons introductory to the modern higher algebra, Hodges and Figgis, Dublin, 1885, (reprinted by Chelsea, New York, 1964).

[W] J. Weyman, The equations of conjugacy classes of nilpotent matrices, Invent. Math. (2) 98 (1989).

Department of Mathematics, Northeastern University, Boston, Massachusetts 02115

E-mail address: weyman@neu.edu 